\title{
Advanced Models in Shock Waves
}

\author{
G. Colonna ${ }^{*, 1}$, G. D’Ammando ${ }^{2}$, A.S. Dikalyuk ${ }^{3}$, M. Panesi ${ }^{4}$, L.D. Pietanza ${ }^{1}$ and S. T. Surzhikov ${ }^{3}$ \\ ${ }^{I}$ CNR-IMIP, Via Amendola 122/D, 70126 Bari, Italy \\ ${ }^{2}$ Dipartimento di Chimica, Universitá degli studi di Bari, Via Orabona 4, 70125 Bari, Italy \\ ${ }^{3}$ A. Ishlinsky Institute for Problems in Mechanics, Russian Academy of Sciences, Prospect Vernadskogo 101-1, Moscow \\ 119526, Russia \\ ${ }^{4}$ University of Illinois at Urbana-Champaign, Urbana, 104 S. Wright Street, 61801 IL, USA
}

\begin{abstract}
The paper presents three collisional-radiative models developed to investigate non-equilibrium chemistry and radiation in hypersonic shock tubes operating with different planetary atmospheres. An hybrid collisional-radiative model, employing the state-to-state kinetics of electronically excited states of molecules and the multi-temperature approximation for the vibrational degree of freedom is presented first, and applied to the numerical rebuilding of experimental shock tube emission spectra. Next, an hybrid collisional-radiative model for ionized air is presented. This model consider the state-tostate approach for electronic states of atoms and the multi-temperature model for the vibrational populations of diatomic molecules in their ground electronic state. A radiative transport equation is also solved to determine radiative source terms in the kinetic scheme and the enthalpy production due to radiation. The third model considers the state-to-state collisionalradiative model of Jupiter's atmosphere, self-consistently coupled with the Boltzmann equation for free electrons and the radiative transfer equation for the radiation transport in one-dimensional slab geometry.
\end{abstract}

Keywords: Collisional-radiative model, radiative transfer, re-entry flows.

\section{INTRODUCTION}

The radiation emitted by the shock-heated gas during the entry of a space vehicle in planetary atmospheres can give a relevant contribution to the heat load of the Thermal Protection System (TPS). In some conditions, the radiative heat flux is effective when the convective and conductive contributions are negligible [1-3].

The flow region downstream a shock wave formed during high-speed atmospheric entry is characterized by a relaxation layer where the gas is far from chemical and thermal equilibrium. The width of this region, ranging from few millimeters to some centimeters, depends on the entry conditions and on the rates of elementary processes acting during the relaxation phase.

Shock tubes [4-8] are devices commonly used to validate the chemical model and the relaxation coefficients of internal degrees of freedom, based on optical emission spectroscopy $[9,10]$. Modeling shock tubes is a fundamental tool for rebuilding experiments. Different approaches are possible to describe the relaxation of internal degrees of freedom and their influence on the chemical processes.

In this work we are reporting three non-equilibrium models describing also the radiation emission. The first model, devoted to the study of non-equilibrium spectral intensity of radiation emitted by the shock-heated gases for

*Address correspondence to this author at the CNR-IMIP, Via Amendola 122/D, 70126 Bari, Italy; Tel: +390805929509; Fax: +390805929520;

E-mail: gianpiero.colonna@ba.imip.cnr.it comparison with available shock tube experimental data, has been developed in the Laboratory of Radiative Gas Dynamics in Moscow [11-13]. After an hybrid collisionalradiative (CR) model coupled to an Euler solver and a radiative transfer equation (RTE), used to investigate the dynamics of the electronic levels of the atoms behind a strong shock wave, has been developed in a joint collaboration between research groups in France and Belgium [14-16]. The third model, a full CR model coupled to an Euler solver, a radiative transfer equation and a Boltzmann equation for the free electrons has been developed in the Plasma Group of CNR in Bari $[17,18]$.

\section{NUMERICAL MODEL}

In this paper we will limit our calculations to stationary shock tubes, which can be described with good accuracy by the 1D Steady Euler equations

$$
\begin{aligned}
& \frac{d \rho u}{d x}=0 \\
& \frac{d}{d x}\left(p+\rho u^{2}\right)=0 \\
& \frac{d}{d x}\left(h+\frac{u^{2}}{2}\right)=\dot{h}
\end{aligned}
$$

where $\rho$ is the mass density, $u$ the flow speed, $p$ the static pressure, $h$ is specific enthalpy. The source term $\dot{h}$ is the enthalpy production rate. Possible contributions can come from electrical discharges (Joule effect), from radiative 
losses or laser heating. The Euler equations need to be coupled with the ideal gas equation of state

$$
\begin{aligned}
& p=\frac{\rho R T}{\bar{M}} \\
& \bar{M}=\sum_{i=1}^{N_{s}} M_{i} \chi_{i}
\end{aligned}
$$

where $T$ is the gas temperature, $R$ is the ideal gas constant, $\bar{M}$ is the mean molar mass and $\chi_{i}=n_{i} / n$ is the molar fraction of the $i$-th species of mass $M_{i}$ and molar density $n_{i}$ ( $n=\sum_{i} n_{i}$ is the total mole density).

The system of equations listed above is not closed because need to define the specific enthalpy. Under Local Thermodynamic Equilibrium (LTE) conditions, the specific enthalpy is a function of the temperature and pressure, $h(T, p)[19-21]$. In shock waves, there is a region, just behind the shock front, in which the LTE assumption is not valid and both chemical and thermal non-equilibrium must be considered. It is necessary to add a kinetic equation for each species

$$
\frac{d \rho_{i} u}{d x}=\dot{\rho}_{i}
$$

where $\dot{\rho}_{i}$ is a source term due to chemical reactions. For the following reaction set $\left(j=1, \ldots, N_{r}\right)$

$$
\sum_{k}^{N_{s}} a_{k j} X_{k} \rightleftharpoons \sum_{k}^{N_{s}} b_{k j} X_{k}
$$

where $X_{k}$ is the $k$-th species and $a_{j k}$ and $b_{j k}$ are the stoichiometric coefficients of the direct and reverse $j$-th reaction.

The chemical reaction source term is given by

$$
\dot{\rho}_{i}=M_{i} \sum_{j=1}^{N_{r}}\left[\left(b_{j i}-a_{j i}\right) k_{j}^{f} \prod_{k=1}^{N_{s}}\left(\frac{\rho_{k}}{M_{k}}\right)^{a_{k j}}+\left(a_{j i}-b_{j i}\right) k_{j}^{r} \prod_{k=1}^{N_{s}}\left(\frac{\rho_{k}}{M_{k}}\right)^{b_{k j}}\right]
$$

The enthalpy per unit mass is given by

$$
h=\frac{1}{\bar{M}} \sum_{i}^{N_{s}} \chi_{i} \bar{H}_{i}
$$

being $\bar{H}_{i}$ the molar enthalpy of the $i$-th species. Under thermal non-equilibrium conditions it is convenient to express the species molar enthalpy as the sum of three contributions

$$
\bar{H}_{i}=\bar{H}_{i}^{T}(T)+\bar{H}_{i}^{i n t}+\bar{H}_{i}^{f}
$$

where the $\bar{H}_{i}^{T}(T)$ term includes translation and those internal degrees of freedom which are assumed in equilibrium with translation. On the other hand $\bar{H}_{i}^{\text {int }}$ is the contribution of internal degrees of freedom which are out of thermal equilibrium and $\bar{H}_{i}^{f}$ is the molar formation energy of the $i$-th species, including the excitation energy of electronically excited states. As a general rule, in evaluating $\bar{H}_{i}^{T}(T)$, only the translational energy is considered for atomic species, while for molecules the rotational degrees of freedom are considered in equilibrium with the translation according to

$\bar{H}_{i}^{T}=\frac{5}{2} R T+\frac{n_{r o t}}{2} R T$

where $n_{r o t}$ is the number of rotational degrees of freedom of the species, e.g. $n_{r o t}=0$ for atoms, 2 for linear molecules and 3 for non-linear molecules.

An important aspect of thermal non-equilibrium kinetics is the dependence of the rate coefficients $\left(k^{f}\right.$ and $k^{r}$ ) in Eq. (5) on the electronic energy states of atoms, and on the electronic and rovibronic energy states of molecules as well as on the temperature of free electrons (for electron impact processes). Selected models, reported in literature $[13,15$, $17,22]$ and briefly discussed in the next sections, differ mainly in the way they consider the influence of internal distributions on the chemical rates [23]. These differences reflect also on the radiation model, since the calculated emissivity and absorption coefficients are directly related to the adopted model for the distribution of population over the available atomic and molecular quantum states, i.e. the internal state distribution (ISD) of each species. For the purposes of this paper, it is useful to distinguish between two approaches which can be used to introduce the effect of excited states in radiative gas dynamics: full and hybrid collisional-radiative models.

In the full collisional-radiative models, all relevant internal states of the species present in the gas mixture and the individual processes linking such states are taken into account. The main difficulties of the full model are the need of large datasets of level-specific cross sections or rate coefficients which, especially for excited states, are not always available, and its high computational cost [15], limiting their application to $1 \mathrm{D}$ or $2 \mathrm{D}$ situations. As a consequence, the full models can hardly be inserted into the 3D CFD codes for complex geometries used in engineering applications.

On the other hand, the hybrid collisional-radiative models attempt to reduce the computational burden using a state-specific description of the electronically excited states which are more important from the point of view of radiation transport and non-equilibrium, while the other degrees of freedom are described by an equilibrium Boltzmann distribution at a given temperature. The multi-temperature approach to thermal non-equilibrium results from associating a different temperature to individual degrees of freedom. A more general multi-internal-temperature approach [24], using several temperatures to describe different energy 
ranges of the ISD of a single degree of freedom, is also possible but it is not considered in this paper.

\section{MULTI-TEMPERATURE KINETICS AND JUST- OVERLAPPING LINE MODEL}

The first investigation in the Laboratory of Radiative Gas Dynamics dedicated to the calculation of non-equilibrium spectral intensity of radiation emitted by the shock-heated gases was presented in 1997 [25]. Since then our group is involved in this area of scientific research [11-13]. In this section, an hybrid collisional-radiative model for the relaxation zone behind a shock is presented. The model solves the Euler system of equations for the determination of flow parameters (see Eqs. (1) and (2)). The equations have been modified in order to take into account reacting gas mixtures following Eqs. (3) to (6). In the calculations presented here, we have neglected in the Euler equations the energy lost by radiation, i.e. $\dot{h}=0$ in Eq. (1). The populations of vibrational degrees of freedom of molecules are calculated introducing a vibrational temperature for each vibrational mode of diatomic and triatomic molecules. The electron gas temperature $T_{e}$ is determined by a separate equation containing different source terms, accounting for the most important electron impact processes.

The following chemical components were accounted in the model: e, N, C, O, $\mathrm{C}_{2}, \mathrm{~N}_{2}, \mathrm{O}_{2}, \mathrm{CN}, \mathrm{CO}, \mathrm{NO}, \mathrm{CO}_{2}, \mathrm{NCO}, \mathrm{C}^{+}, \mathrm{N}^{-}$, $\mathrm{O}^{+}, \mathrm{C}_{2}^{+}, \mathrm{N}_{2}^{+}, \mathrm{O}_{2}^{+}, \mathrm{CN}^{+}, \mathrm{CO}^{+}, \mathrm{NO}^{+}$. The description of the transformations among the chosen chemical species was performed according to the chemical kinetic mechanism based on the results of the studies [26-32]. The populations of the radiating electronic states of molecules, which are considered as independent chemical species in the model [33], are calculated by solving the corresponding kinetic equations. The following molecular electronically excited states have been considered: $\mathrm{CN}\left(A^{2} \Pi\right), \mathrm{CN}\left(B^{2} \Sigma^{+}\right), \mathrm{C}_{2}\left(d^{3} \Pi\right), \mathrm{CO}\left(A^{1} \Pi\right), \mathrm{NO}\left(A^{2} \Sigma^{+}\right)$, $\mathrm{NO}\left(B^{2} \Pi\right), \mathrm{NO}\left(C^{2} \Pi\right), \mathrm{N}_{2}\left(A^{3} \Sigma\right), \mathrm{N}_{2}\left(B^{3} \Pi\right), \mathrm{N}_{2}\left(C^{3} \Pi\right)$, $\mathrm{N}_{2}^{+}\left(B^{2} \Sigma\right)$. The mechanism of population of these states includes the following processes: excitation/de-excitation of the electronic states of molecules by electron impact, spontaneous emission, excitation/de-excitation of the electronic states of molecules in collisions with heavy particles. The data needed to model these processes were partially taken from [30, 34-36]. The effect of the non-equilibrium dissociation was accounted using the Kuznetsov model [37]. It is worth noticing that the results of the experimental measurements of the nonequilibrium radiation of the shock-heated $\mathrm{CO}_{2}-\mathrm{N}_{2}$ and $\mathrm{N}_{2}-\mathrm{O}_{2}$ gas mixtures were successfully reconstructed using the same set of parameters of the model without any special adjustment (it means the same set of chemical reactions rates and rates of the processes of population of electronic excited states of molecules).

In the calculation of enthalpy, molecular rotation is considered in equilibrium with the translation, therefore the ro-translational contribution to the energy is that given in Eq. (8) while the internal enthalpy considers the vibrational contribution of each electronic state
$\bar{H}_{i}^{\text {int }}=\sum_{j=1}^{N_{v, i}} g_{i, j} \frac{R \theta_{i, j}}{\exp \left(\theta_{i, j} / T_{v, i, j}\right)-1}=\sum_{j=1}^{N_{v, i}} M_{i} g_{i, j} e_{i, j}\left(T_{v, i, j}\right)$

Here $T_{v, i, j}, \theta_{i, j}, g_{i, j}$ are respectively the vibrational temperature, the characteristic vibrational temperature and the degeneracy of $j$-th vibrational mode of $i$-th molecule (which can be an electronically excited state), $N_{v, i}$ is the number of vibrational modes of $i$-th molecule and $e_{i, j}\left(T_{v, i, j}\right)$ is the vibrational energy per unit mass at the given vibrational temperature.

To determine the vibrational temperatures, we consider the following relaxation equation

$$
\frac{d u e_{s, j}\left(T_{v, s, j}\right)}{d x}=Q_{s, j}^{V T}+Q_{s, j}^{C V}
$$

where $Q_{s, j}^{V T}$ takes into account the vibration-translation energy transfer and $Q_{s, j}^{C V}$ the influence of chemical processes on the vibrational energy. On the other hand, the $Q_{s, j}^{V V^{\prime}}$ term, modeling the vibration-vibration energy exchange has been neglected in the present radiation model, for the following reason. In [29] it was shown that in relatively strong shock wave the $V-V$ exchange influences only on the relative values of the vibrational temperatures. Under the present conditions $\left(p \sim 1\right.$ torr, $\left.v_{s h} \sim 5-8 \mathrm{~km} / \mathrm{s}\right)$ it is very unlikely that the multi-temperature approach could correctly determine the vibrational distribution function of the molecules at each point behind the shock front. On the other hand, if the aim is the calculation of the radiation of shockheated gases, then in our opinion the most important factor is the correct modeling of the kinetics of electronically excited states: the influence of the vibrational temperature on the radiation is a second order effect. CV processes have a very important effect on the vibrational temperature, and accounting for that process could change significantly (several thousand $\mathrm{K}$ ) the profile of the vibrational temperature behind the shock front. Clearly, in the absence of intensive chemical transformations it is necessary to include the $V-V^{\prime}$ mechanism in the model.

The $Q_{s, j}^{V T}$ source terms are calculated in the LandauTeller approximation as

$Q_{s, j}^{V T}=\frac{e_{s, j}(T)-e_{s, j}\left(T_{v, s, j}\right)}{\tau_{s, j}}$

where the relaxation times $\tau_{s, j}$ are usually estimated using the Millikan-White approximations combined with Park's correction. The influence of chemical kinetics and vibrational relaxation is taken into account in the model (CVDV interaction). The following equation is used for the determination of the temperature of electron gas

$$
\frac{d}{d x}\left(\frac{3}{2} T_{e} X_{e} u\right)+\frac{3}{2} T_{e} X_{e} \frac{d u}{d x}=Q_{e i}+Q_{e a}+Q_{a i}+Q_{i o n}+Q_{e v}
$$


The rates of the processes responsible for the variation of the free electron temperature $T_{e}$ are described by the following $Q$-terms: elastic electron-ion ( $e i)$ and electronatom ( $e a$ ) collisions; associative ionization/dissociative recombination ( $a i$ ); ionization (ion ); interaction of electron gas and vibrational modes of molecules (ev). The term $Q_{e e}$, corresponding to electron impact excitation/de-excitation of molecules and ions, has been omitted in Eq. (12), since in the moderate intensity shock waves investigated by the present model (the degree of ionization is less than $0.2 \%$ in air at $v_{s h} \leq 8 \mathrm{~km} / \mathrm{s}$ ), the population of excited state of molecules is much less than the density of free electrons, resulting in a negligible effect of electron impact excitation processes on $T_{e}$.

The just-overlapping line model [38] is used for the spectral intensity of radiation calculations. The equations of the model are as follows $\left(\mathrm{Wcm}^{-3} \mu \mathrm{m}^{-1} \mathrm{sr}^{-1}\right)$

$$
\begin{aligned}
j_{\lambda} & =3.20210^{-10} \frac{N_{e e l}}{Q_{V R} \lambda^{6}} \sum_{V^{\prime}, V^{\prime \prime}} \frac{S_{V^{\prime} V^{\prime \prime}}}{\Delta B_{V} \mid} \exp \left[-\frac{h c}{k T_{v}} E_{e e l}\left(V^{\prime}\right)\right. \\
& \left.-\frac{h c}{k T_{R}} \frac{B_{V^{\prime}}}{\Delta B_{V}}\left(\omega-\omega_{V^{\prime} V^{\prime \prime}}+B_{V^{\prime}}\right)\right]
\end{aligned}
$$

where $N_{e e l}\left(\mathrm{~cm}^{-3}\right)$ is the population of the electronic excited state of the molecule; $\omega\left(\mathrm{cm}^{-1}\right)$ is the wave number; $\lambda(\mathrm{nm})$ is the wavelength; $\omega_{V^{\prime}, V^{\prime \prime}}\left(\mathrm{cm}^{-1}\right)$ is the wavenumber of a vibronic transition; $\Delta B_{V}=B_{V^{\prime}}-B_{V^{\prime \prime}}$ and $B_{V^{\prime}}, B_{V^{\prime \prime}}$ are the rotational constants of the upper and lower molecular electronic states respectively; $Q_{V R}$ is the rovibrational partition function of the electronic excited state of the molecule; $S_{V^{\prime}, V^{\prime \prime}}$ the strength of the vibronic transition; $E_{e e l}\left(V^{\prime}\right)$ is the energy of the vibrational level $V^{\prime}$ of the electronic excited state; $T_{V}, T_{R}$ are the vibrational and rotational temperatures. $S_{V^{\prime}, V^{\prime \prime}}$ is determined from the square of the moment of the electronic transition by the following formulas

$$
\begin{aligned}
& S_{V^{\prime}, V^{\prime \prime}}=\left(2-\delta_{0, \Lambda^{\prime}+\Lambda^{\prime \prime}}\right)(2 S+1)\left|R_{V^{\prime}, V^{\prime \prime}}\right|^{2} \\
& \left|R_{V^{\prime}, V^{\prime \prime}}\right|^{2}=\frac{3 h}{64 \pi^{4} a_{0}^{2} q_{e}^{2}} \frac{2-\delta_{0, \Lambda^{\prime}}}{2-\delta_{\Lambda^{\prime}+\Lambda^{\prime \prime}}} \frac{1}{\omega_{V^{\prime}, V^{\prime \prime}}^{3}} A_{V^{\prime}, V^{\prime \prime}}
\end{aligned}
$$

where " $\Lambda$ " is the quantum of the projection of the orbital angular momentum on the internuclear axis. $A_{V^{\prime}, V^{\prime \prime}}$ is the Einstein coefficient of vibronic transition that can be obtained as a result of solution of quantum mechanical problem of the calculation of vibronic wave functions.

The full description of the model can be found in [11-13, 25]. It is worth noting that what is presented here is actually a framework: different gas mixtures and chemical kinetics models can be studied by this code, with the possibility of studying both non-equilibrium and equilibrium spectral intensities of radiation emitted by shock-heated gases.

The second part of activity in our group is dedicated to the systematic processing of experimental data obtained on different shock tube facilities all around the world by means of the model developed in our group. Up to now we have performed the analysis of results obtained on the shock tubes of NASA Ames Research Center (EAST facility [4,5], Chofu Aerospace Center [6], Queensland University (X2 facility) [7], Institute of Mechanics Moscow State University [8]. By means of our model we have studied spectral intensity of radiation of shock-heated $\mathrm{N}_{2}-\mathrm{O}_{2}, \mathrm{CO}_{2}-\mathrm{N}_{2}, \mathrm{CH}_{4}-\mathrm{N}_{2}$ gas mixtures.

Figs. (1, 2) report a comparison between numerical results calculated by the present model (dashed line) and experimental data (solid line). In Fig. (1), the nonequilibrium spectral intensity of the radiation of an $80 \% \mathrm{~N}_{2}-$ $20 \% \mathrm{O}_{2}$ gas mixture at $p=1$ torr heated by a shock wave with velocity $v_{s h}=5.56 \mathrm{~km} / \mathrm{s}$. The most important electronic band systems generating the observed radiation in this spectral region are: $\mathrm{N}_{2} 2^{\text {nd }}$ positive $\left(C^{3} \Pi-B^{3} \Pi\right), \mathrm{N}_{2}^{+} 1^{\text {st }}$ negative $\left(B^{2} \Sigma-X^{2} \Sigma\right)$, NO $\gamma$-system $\left(A^{2} \Sigma-X^{2} \Pi\right)$, NO $\beta$ -system $\left(B^{2} \Pi-X^{2} \Pi\right)$ and NO $\delta$-system $\left(C^{2} \Pi-X^{2} \Pi\right)$.

For the $\mathrm{N}_{2}-\mathrm{O}_{2}$ gas mixture, the reported spectral distribution is typical in the 200-400 nm spectral region at shock wave speeds $5 \mathrm{~km} / \mathrm{s} \leq v_{s h} \leq 8 \mathrm{~km} / \mathrm{s}$. As the shock wave speed increases, the intensity of non-equilibrium radiation in the spectral range $300-400 \mathrm{~nm}$, mainly produced by the $\mathrm{N}_{2} 2^{\text {nd }}$ positive and $\mathrm{N}_{2}^{+} 1^{\text {st }}$ negative band system systems, increases faster than the spectral intensity of radiation in the 200-300 nm range generated by the NO $\gamma, \beta$ and $\delta$ - systems. On the other hand, in the whole spectral range $(200-400 \mathrm{~nm})$ the radiation intensity increases with the shock velocity. The model successfully describes the above discussed trends in the experimental data.

In Fig. (2) the non-equilibrium spectral intensity of the radiation of $70 \% \quad \mathrm{CO}_{2}-30 \% \quad \mathrm{~N}_{2}$ gas mixture at pressure $p=0.3$ torr heated by the shock wave with velocity $v_{s h}=6.76 \mathrm{~km} / \mathrm{s}$ is reported. The main radiative electronic band systems in this case are $\mathrm{CO} 4^{\text {th }}$ positive $\left(A^{1} \Pi-X^{1} \Sigma^{+}\right)$, NO $\gamma$-system $\left(A^{2} \Sigma^{+}-X^{2} \Pi\right)$, NO $\beta$-system $\left(B^{2} \Pi-X^{2} \Pi\right)$, NO $\delta$-system $\left(C^{2} \Pi-X^{2} \Pi\right.$, active between 200 and $340 \mathrm{~nm}), \mathrm{CN}$ violet $\left(B^{2} \Sigma^{+}-X^{2} \Sigma^{+}\right.$, active between 340 and $440 \mathrm{~nm}), C_{2}$ Swan $\left(d^{3} \Pi-a^{3} \Pi\right.$, active between 440 and $560 \mathrm{~nm})$ and $\mathrm{CN}$ red $\left(A^{2} \Pi-X^{2} \Sigma^{+}\right.$, active between 560 and $800 \mathrm{~nm})$. NO molecules play a more important role in the formation of the radiation in ultraviolet part of the spectrum due the higher amount of $\mathrm{N}_{2}$ molecules in the test gas mixture compared to the actual composition of the Mars atmosphere, which is $96 \% \mathrm{CO}_{2}-4 \% \mathrm{~N}_{2}$.

In [39] the error in the measured spectral intensity of radiation was estimated to be $90 \%$. Taking into account this 
value agreement between experimental and numerical data should be regarded as acceptable.



Fig. (1). Non-equilibrium spectral emissivity of the relaxation zone behind the shock wave initiated in $80 \% \mathrm{~N}_{2}-20 \% \mathrm{O}_{2}$ gas mixtures at $P=1$ Torr, $V_{s h}=5.56 \mathrm{~km} / \mathrm{s}$.



Fig. (2). Non-equilibrium spectral emissivity of the relaxation zone behind the shock wave initiated in $30 \% \mathrm{~N}_{2}-70 \% \mathrm{CO}_{2}$ gas mixtures at $P=0.3$ Torr, $V_{s h}=6.76 \mathrm{~km} / \mathrm{s}$.

\section{HYBRID COLLISIONAL RADIATIVE MODELING OF THE FIRE II FLIGHT EXPERIMENT}

The results of experimental and numerical analyses of the aerothermal environment surrounding space capsules entering Earth's atmosphere put forward the key role played by ionization to post-shock relaxation $[15,40]$. The convective and radiative components of heat-flux generated during the atmospheric reentry are affected by the ionization degree. The ionization process is initiated by the associative ionization $\mathrm{N}+\mathrm{O} \rightarrow \mathrm{NO}^{+}+\mathrm{e}^{-}[41,42]$ thanks to relatively low activation energy of this process and also because it does not involve charged particles. When a sufficient number of electrons is generated, the ionization is favored by electron impact processes. Thus, to model accurately the evolution of the ionization degree it is necessary to account for the kinetics of atom formation (e.g. dissociation [43-45]). Another important contribution to ionization can come from excited atoms, which enhance the electron production in high enthalpy flows. The discussion which follows focuses on the consistent modeling of the interaction between chemistry and radiation. In literature, the coupling of these processes is often approximately accounted for in crude manner by using escape factors. In this work, in order to correctly describe coupling between radiation and excited atoms, the species conservation equations are directly coupled with the radiative transfer equation. Results are discussed for the flow surrounding the Fire II flight experiment.

\subsection{Transport Equations}

This section discusses the model used to describe the dynamics of multi-component flowing air considering chemical reactions, relaxation of atomic levels and radiation transport.

Here we are accounting for 95 species, considering separately electronic levels of atomic species, providing the internal distribution of atoms, listed in [46] and the molecular species $\mathrm{N}_{2}, \mathrm{NO}, \mathrm{O}_{2}, \mathrm{~N}_{2}^{+}, \mathrm{NO}^{+}, \mathrm{O}_{2}^{+}$. On the other hand, the vibrational distribution of molecules is assumed to follow the Boltzmann statistic, all with the same temperature $\mathrm{T}_{\mathrm{v}}$, while their rotation is considered in equilibrium at the gas temperature.

\subsubsection{Thermodynamics}

Air mixture is composed by $\mathrm{O}_{2}$ and $\mathrm{N}_{2}$ and related species such as, NO, N with 46 levels, $\mathrm{O}$ with 40 levels, $\mathrm{N}_{2}^{+}$, $\mathrm{O}_{2}^{+}, \mathrm{NO}^{+}, \mathrm{N}^{+}, \mathrm{O}^{+}$, and $\mathrm{e}^{-}$. The atomic levels can be actual states or lumped levels, obtained by grouping states with similar energies. The thermodynamic properties of the molecular and ionic species are calculated following the Gurvich tables [47].

\subsubsection{Shock Tube Flow Solver}

A one-dimensional solver, discussed in several articles [14-16], was written to simulate shock heated plasma flows. More recently, this model has been updated to simulate highspeed Earth entry conditions. Firstly, the additional source term $Q_{\text {Rad }}$ has been included to account for the radiation transport in the continuity equation of the energy. Secondly, the contribution of the radiation transfer is also included in the kinetics of atomic levels. Post-shock conditions are computed by using the Rankine-Hugoniot equations, freezing both the composition, the internal distributions and internal temperatures. The evolution of the system after the shock is calculated by solving the stationary Euler equations in space marching approach, closed by the continuity equation for each generalized species and the evolution equations for the vibrational and electron temperatures. Finally, the radiation field is determined by solving the Boltzmann equation for the photon. 
Compared to the general formulation of section 2, the present model has the following characteristics:

- the enthalpy production term $\dot{h}$ of Eq. (1) is modified to explicitly include the contributions of radiation as

$$
\dot{h}=-\frac{\partial Q_{r a d}}{\partial x}
$$

- $\quad$ additional vibrational energy conservation equations including the radiative losses from each vibrational mode are solved

$$
\frac{\partial}{\partial x}\left(\rho u e_{m}\right)=\Omega^{m}-\frac{\partial}{\partial x} Q_{R a d}^{m}+\cdots, \quad[m \in \mathcal{U}]
$$

- the following radiative transfer equation in slab geometry is solved

$$
\frac{\mu}{\kappa_{\lambda}} \frac{d I_{\lambda}}{d x}+I_{\lambda}(x)=\frac{\eta_{\lambda}}{\kappa_{\lambda}}(x)=S_{\lambda}(x)
$$

where the flow velocity is indicated with $u$. In Eq. (16), $e_{m}$ represents the energy of the vibrational modes, when referred to molecules, and to translational energy, when referred to free electrons, while the corresponding source term due to chemical processes and VT and VV relaxation collisions is $\Omega^{m}$.

Eq. (17) describes the evolution of the spectral intensity $\left(I_{\lambda}\right)$ in a given direction $\vartheta(\mu=\cos \vartheta)$ with respect to the flow direction, given the emissivity $\left(\eta_{\lambda}\right)$ and the spectral absorption $\left(\kappa_{\lambda}\right)$ coefficients, which ratio is $S_{\lambda}$.

\subsection{Kinetic and Radiative Processes}

The solution of the equations discussed in the previous section requires a detailed data set for rate coefficients and spectroscopic data. Different collisional-radiative models have been developed to study the kinetics in air for $0 \mathrm{D}$ calculations [48-50]. In this work we have used the Bultel et al. [48] database, improved by adding new cross section data for internal excitation by electron impact and dissociative recombination rates of $\mathrm{NO}^{+}, \mathrm{O}_{2}^{+}$and $\mathrm{N}_{2}^{+}$.

The theory governing the dynamics of photons Eq. (17), chemical species and energy relaxation Eq. (16) is discussed in details in [14].

\subsubsection{FIRE II Flight Experiment}

The main objective of this work is to gain insights on the behavior of the atomic excited states in strong nonequilibrium flows. The flow conditions are taken from the trajectory of a famous flight experiment, FIRE II, mainly intended to characterize the heating fluxes during high speed entry [14].

\subsubsection{Composition and Flow Field}

The characterization of the plasma formed after the shock front needs to determine accurately the density of the species and the distribution of internal states.
Collisions redistribute the energy among the different degrees of freedom, i.e. vibrational and electronic states and chemical reactions, affecting the spatial profile of translational and vibrational temperatures as depicted in Fig. (3a).

The vibrational temperature, $T_{v}$, increases due to VT energy transfer, until the onset of chemical processes, enhanced by the vibrational excitation. The vibrational energy loss due to dissociation equilibrate the vibrational pumping, reaching a quasi-steady state.

The temperature profile is affected also by the radiation model, determining the energy lost by the system due the photon escape. The sensitivity to optical characteristic of the plasma is demonstrated in Fig. (3b), where the electron density profile calculated with the coupled model here described is compared with the optically thick, i.e. complete radiation reabsorption, and thin, i.e. fully transparent plasma, approximations. In the thick case, the equilibrium is rapidly established, while in the thin case, the radiation tends to depopulate the excited levels, thus delaying both excitation and ionization. In this last case the electron density profile exhibits a maximum, rapidly decaying due to energy lost by radiation. The coupled model is in agreement with the optically thick case.

In the considered conditions, the average electron energy is relatively low and electrons are unable to ionize the $\mathrm{N}$ and $\mathrm{O}$ atoms in their ground states and low-lying metastable states.

Ionization is promoted by low-threshold process initiated from electronically excited atoms and therefore two-step excitation-ionization is favored with respect to direct ionization from the ground state, thus to accurately determine the electron density is necessary to determine the distribution of internal levels, that can depart from the Boltzmann distribution.

To this aim, in Fig. (4), the Boltzmann distribution (at $T_{e}$ ) at $1 \mathrm{~cm}$ from the shock front is compared with the distributions of nitrogen (Fig. 4a) and oxygen (Fig. 4b) calculated according to the different radiation models. The results show that the low-lying states present a different evolution with respect to highly excited ones. In fact, while low-energy states are well approximated by the Boltzmann distribution at $T_{e}$, because of small ionization rates, the high lying states are underpopulated, as a consequence of their rapid ionization.

\section{COLLISIONAL-RADIATIVE MODEL OF $\mathrm{H}_{2} / \mathrm{He}$ PLASMA FOR ENTRY IN JUPITER ATMOSPHERE}

The non-equilibrium models based on multi-temperature approach assume that the internal states are described by a Boltzmann distribution and the free electrons follow the Maxwell-Boltzmann distribution function. In order to evaluate the energy content of the different degrees of freedom, the multi-temperature approach gives good accuracy, because, in most practical cases, the low energy distribution follow the Boltzmann function. However it frequently occurs that the highenergy tail of the distributions strongly departs from equilibrium as a consequence of the coupling between internal states and 


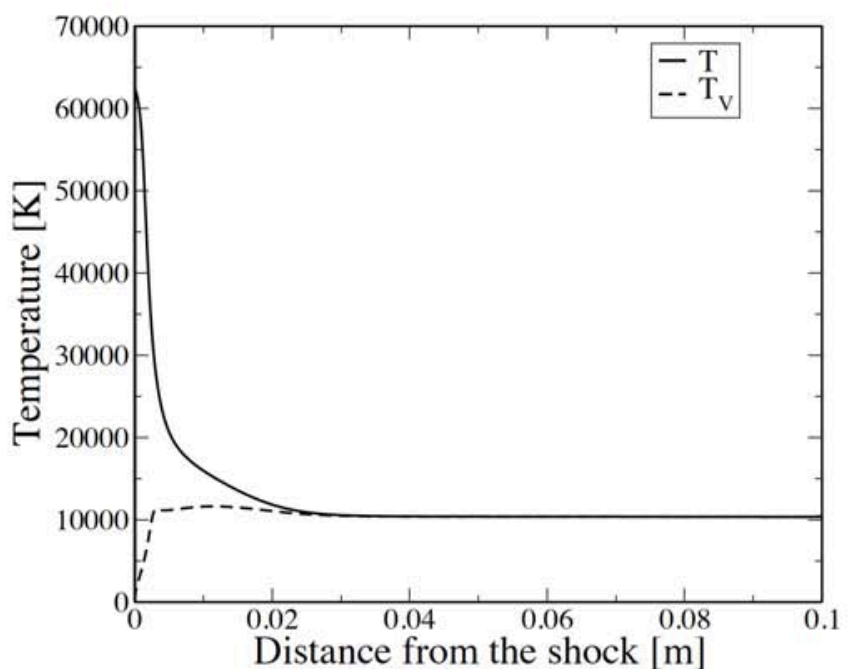

(a) Post shock temperature profile.

Fig. (3). Temperature and electron density profiles in the post shock.

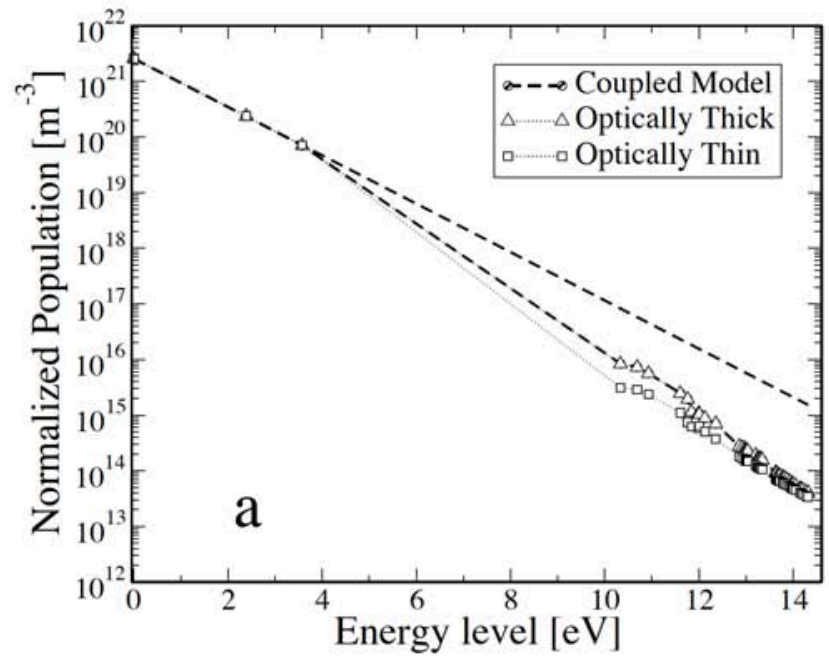

(a) Electronic distribution of nitrogen.

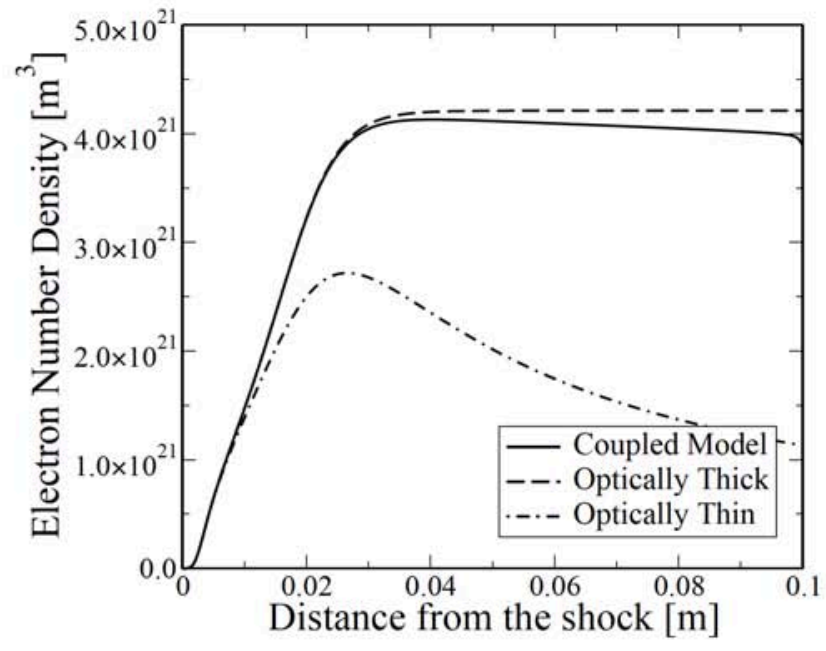

(b)Elesctron density as a function of the optical thickness.

Fig. (4). Population distributions of atomic species at $1 \mathrm{~cm}$ from the shock. The equilibrium distribution of the electronic levels is indicated in dashed line. The self-consistent model is indicated in circles, while the triangles and the square symbols refers to optically thick and optically thin approximation respectively.

chemical processes. This is a crucial aspect because exothermic processes are strongly favored from excited states and, as a consequence, the rate coefficients depart from the Arrhenius trend.

These aspects can be investigated using the full state-to-state model presented in this section. This model solves the master equation for chemical kinetics considering transitions between internal states and chemical processes involving excited levels. The main differences between the model presented in this section and the previously presented models are:

- The population distribution over excited states of atoms and molecules are determined solving a kinetic master equation system including the most significant populating/depopulating processes for each internal state. The multi-temperature approximation is not invoked for any internal degree of freedom.

- Due to the strong coupling between free electrons and excited states of heavy particles, it is not assumed that free electrons follow a Maxwell-Boltzmann distribution function. Instead, to properly describe the time dependence of the electron energy distribution (eedf), the Boltzmann equation for free electron must be solved.

In this case, strongly non-equilibrium eedf can be obtained, characterized by a rich structure (peaks, bumps, rapid change of slope, plateaux), due to electron collisions with atoms and molecules, reflecting the structure of the corresponding cross sections. An important contribution comes from superelastic collisions, where the energy is 
transferred from excited states of atoms and molecules to electrons, increasing the high-energy tail of the distribution. There is a strict correlation between internal level populations and the eedf: the distribution of internal level is input data for the Boltzmann distribution, while the rate coefficients of electron impact induced processes enter in the master equation for the plasma composition. These rates are calculated as

$$
K_{e}=\int_{0}^{\infty} \sqrt{\varepsilon} f_{e}(\varepsilon) \sigma(\varepsilon) v_{e}(\varepsilon) d \varepsilon
$$

where $\varepsilon$ is the electron energy, $f_{e}$ is the eedf normalized in the energy space, $v_{e}$ is the relative velocity and $\sigma$ is the process cross section.

To complete the kinetic model, the interaction with radiation should be considered, accounting for photon emission and absorption. This problem requires to couple the kinetic equation with the radiation (collisional-radiative model) considering also an equation for the photon transport, to determine the absorption coefficients. The strict connection between chemical kinetics, electron kinetics, radiation transport and fluid dynamics makes this problem strongly non-linear, and requires a self-consistent approach which solves at the same time all the equations, as schematized in Fig. (5).

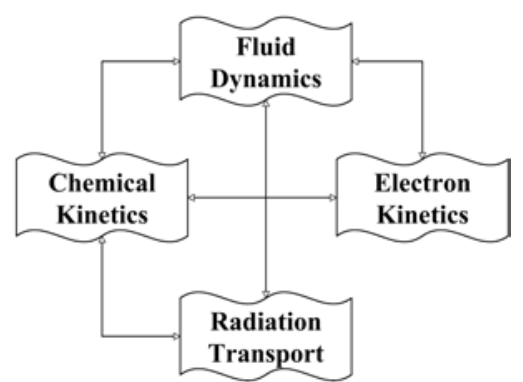

Fig. (5). Schematic plot of the self-consistent approach coupling fluid dynamics, chemical and electron kinetics and radiation transport.

The self-consistent approach, without radiation transport, has been applied to nozzle expansion for different mixtures [51-53], also in presence of applied electric and magnetic fields $[54,55]$, in the boundary layer of hypersonic vehicle $[56,57]$, a 2D flow around a cylinder [58] and in gas discharges [59], neglecting the radiation. Recently, a full model also including radiation transfer is being developed and applied to 1D shock waves in hydrogen [17] and hydrogen-helium (Jupiter atmosphere) mixtures [18].

\subsection{State-to-State Kinetics}

The internal energy given in Eq. (6) has been calculated solving a continuity equation for each species in each internal state analogous to Eq. (3) where $\dot{\rho}_{i}$ is the source term accounting for the production/consumption of an internal level of a chemical specie due to internal transitions and chemical reactions [51,53].

The present $\mathrm{CR}$ model for atomic $\mathrm{H} / \mathrm{He}$ considers electron induced transition and ionization/three body recombination

$$
\begin{aligned}
& A(n)+e^{-} \rightleftharpoons A(m)+e^{-} \\
& A(n)+e^{-} \rightleftharpoons A^{+}+2 e^{-}
\end{aligned}
$$

the radiative losses

$$
\begin{aligned}
& A(n) \rightarrow A(m<n)+h v_{m n} \\
& A(m<n)+h v_{m n} \rightarrow A(n) \\
& A^{+}+e^{-} \rightarrow A(n)+\rightsquigarrow
\end{aligned}
$$

where $\mathrm{A}=\mathrm{H}, \mathrm{He}$. Details of the $\mathrm{CR}$ model for $\mathrm{H}$ atom and corresponding cross sections can be found in [60], while $\mathrm{He}$ atom has recently been introduced to study atmospheric entry in Jupiter atmosphere [18], closely following [61,62] for the construction of the level energies, and using statespecific electron impact cross sections reported in [63]. The transition probabilities of He have been taken from NIST's online database [64] while radiative-recombination cross sections have been determined from Opacity Project's [65] theoretical photoionization cross-section using Milne's [66] relation.

For $\mathrm{H}$ atoms we also include the atom-atom excitation and ionization processes $[67,68]$

$$
\begin{aligned}
& H(n)+H(1) \rightleftharpoons H(m)+H(1) \\
& H(n)+H(1) \rightleftharpoons H^{+}+H(1)+e^{-}
\end{aligned}
$$

which are fundamental in the region just behind the shock front to produce the first electrons, so that the electron induced transitions become effective. Differently from traditional CR models, where the radiation absorption was considered by the way of the escape factor, we include separately photon emission, both spontaneous and stimulated, and absorption, calculated by the radiation transport module.

The model has been completed by adding the vibrational, dissociation and ionization kinetics of hydrogen molecules in the ground electronic state, induced either by moleculemolecule and atom-molecule collisions

$$
\begin{aligned}
& H_{2}(v)+H_{2}(w) \rightleftharpoons H_{2}(v+1)+H_{2}(w-1) \\
& H_{2}(v)+H_{2} \rightleftharpoons H_{2}(w)+H_{2} \\
& H_{2}(v)+H \rightleftharpoons H_{2}(w)+H \\
& H_{2}(v)+H_{2} \rightleftharpoons 2 H+H_{2} \\
& H_{2}(v)+H \rightleftharpoons 2 H+H
\end{aligned}
$$

The considered electron impact processes are: resonant vibrational excitation, ionization and dissociation for $\mathrm{H}_{2}$ and ionization, dissociation and dissociative recombination for $\mathrm{H}_{2}^{+}$

$$
\begin{aligned}
& H_{2}(v)+e^{-} \rightleftharpoons H_{2}(w)+e^{-} \\
& H_{2}(v)+e^{-} \rightleftharpoons e^{-}+H(n=1)+H(n=1,2,3) \\
& H_{2}(v)+e^{-} \rightleftharpoons H+H^{+}+2 e^{-} \\
& H_{2}^{+}+e^{-} \rightleftharpoons 2 H^{+}+2 e^{-} \\
& H_{2}^{+}+e^{-} \rightleftharpoons H+H^{+}+e^{-} \\
& H_{2}^{+}+e^{-} \rightleftharpoons 2 H
\end{aligned}
$$

The next subsection presents results of our model [69], under conditions similar to those met by a space vehicle entering the atmosphere of Jupiter at hypersonic speed. We 
consider both optically thin and optically thick conditions at an entry speed of $M=20$.

\subsection{Mach 20}

It is assumed that Jupiter'a atmosphere is composed by $\mathrm{H}_{2}$ and $\mathrm{He}$ in the ratio 89:11. For the shock tube simulation we impose the free stream flow conditions and chemical composition as follows: $T_{0}=160 \mathrm{~K}, P_{0}=10 \mathrm{~Pa}$, corresponding to a free stream gas flow speed $v_{0} \approx 18.4 \mathrm{Km} / \mathrm{s}$, with initial Boltzmann distributions at $160 \mathrm{~K}$ for $\mathrm{H}_{2}, \mathrm{He}$ and initial Maxwell-Boltzmann distribution at $160 \mathrm{~K}$ for the free electrons.

Behind the shock the gas temperature raises to $T_{g}=13,000 \mathrm{~K}\left(P=4.610^{3} \mathrm{~Pa}\right)$, inducing both chemical reactions and redistribution of the populations over the internal states of atoms and molecules. In Fig. (6a) the postshock composition profiles of the different species are reported as a function of shock distance, showing the formation of an highly dissociated, weakly ionized plasma in the range of distances $0.1-1 \mathrm{~m}$.

In Fig. (6b) the evolution of temperatures associated to different degrees of freedom, i.e. translational for the gas and the free electrons and internal for the molecules and atoms is reported. The gas temperature $T_{g}$ is directly obtained from the translational enthalpy, as a part of the solution algorithm of the Euler equations, while the electron temperature is calculated as $T_{e}=\frac{2 \bar{E}_{e}}{3 k}$ where $\bar{E}_{e}$ is the average translational electron energy associated to the non-equilibrium eedf. On the other hand, the internal temperatures $T_{H}$ and $T_{H e}$ are determined interpolating with a Boltzmann distribution the populations of the ground and first excited state of the atoms, and of the $v=0$ and $v=1$ vibrational level populations for $\mathrm{H}_{2}$ as obtained from the CR model. Internal temperatures, which essentially provide an estimate of the energy stored in the low energy part of the internal state distributions of atoms and molecules, are useful to compare the results of the present detailed state-to-state models and the multitemperature models. The figure shows a smooth convergence of $T_{g}, T_{e}$ and $T_{H_{2}}$ to the same limit value as a result of the strong coupling between translation and vibrational degrees of freedom due to both heavy particle and electron impact processes, while the internal temperature of the $\mathrm{H}$ and $\mathrm{He}$ atoms present a non-monotone behavior.

In Fig. (7a) the evolution of the internal distribution of atomic hydrogen is reported as a function of the shock distance, showing a strong deviation from the equilibrium distribution at small distances from the shock, approaching a Boltzmann distribution at large distances. The evolution of the eedf is reported in Fig. (7b), and shows a significant deviation from the Maxwellian distribution at both small and high distance from the shock. Specifically, the eedf is characterized by plateaux induced by the superelastic collisions between low-energy electrons and $\mathrm{H}(n=2)$ atoms, this effect being more important at relatively low electron temperature, while at higher $T_{e}$ the plateaux tend to reduce even if the action of superelastic collisions is still quite large.

The understand the effect of different radiation models, we have compared (a) a self consistent approach with radiation transfer coupled to chemical kinetics through the RTE; (b) a simplified approach where radiation transfer is neglected, i.e. the plasma is considered optically thin or thick to radiation. We have reported in Fig. (8a) the molar fraction of the $\mathrm{H}(n=2)$ excited hydrogen atom in the post-shock


Fig. (6). Post-shock molar fraction and temperature profiles as a function of the distance $d$ from the shock front for $M=20$. 

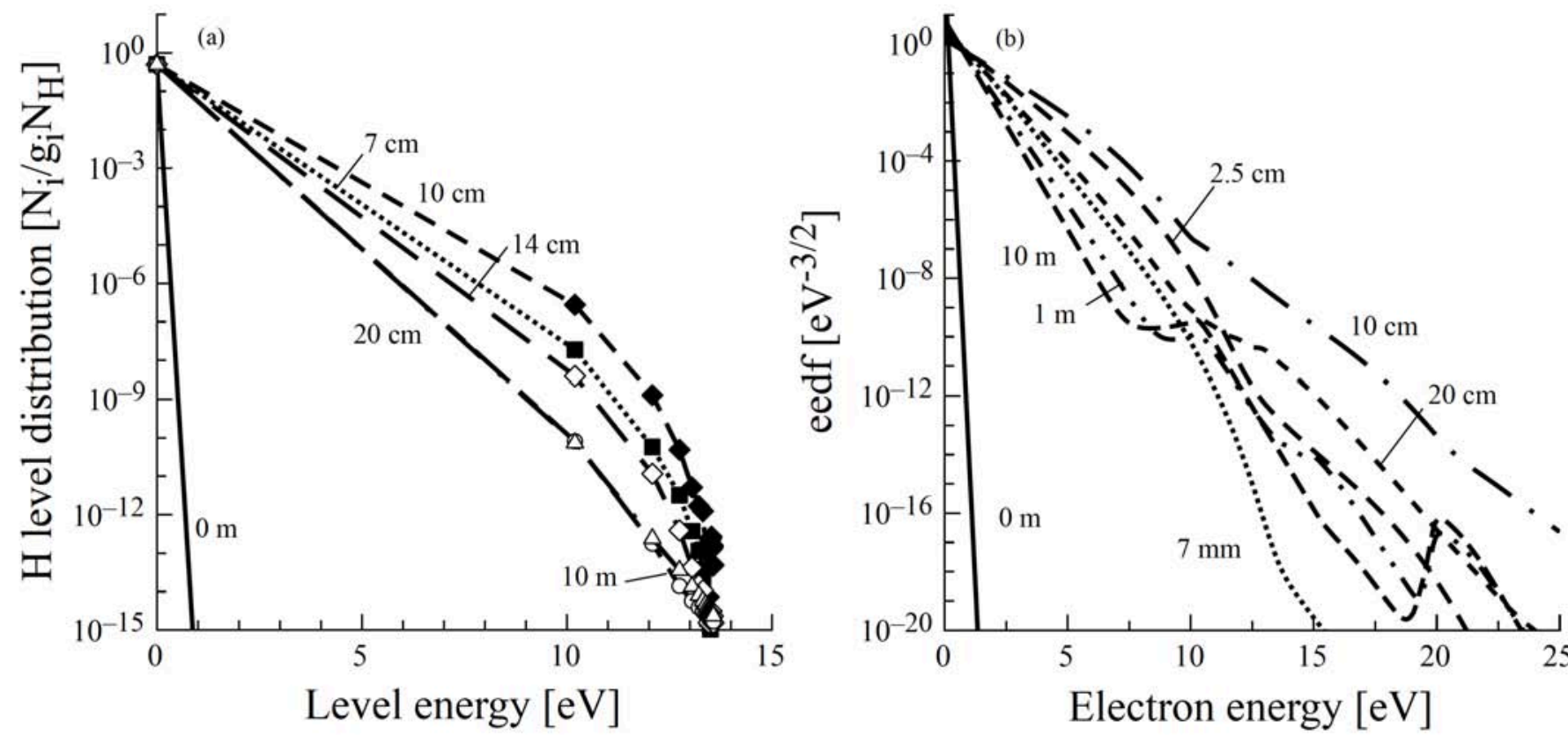

Fig. (7). (a) ISD of $\mathrm{H}$ atom and (b) eedf in the $\mathrm{H}_{2} / \mathrm{He}$ plasma for $M=20$.
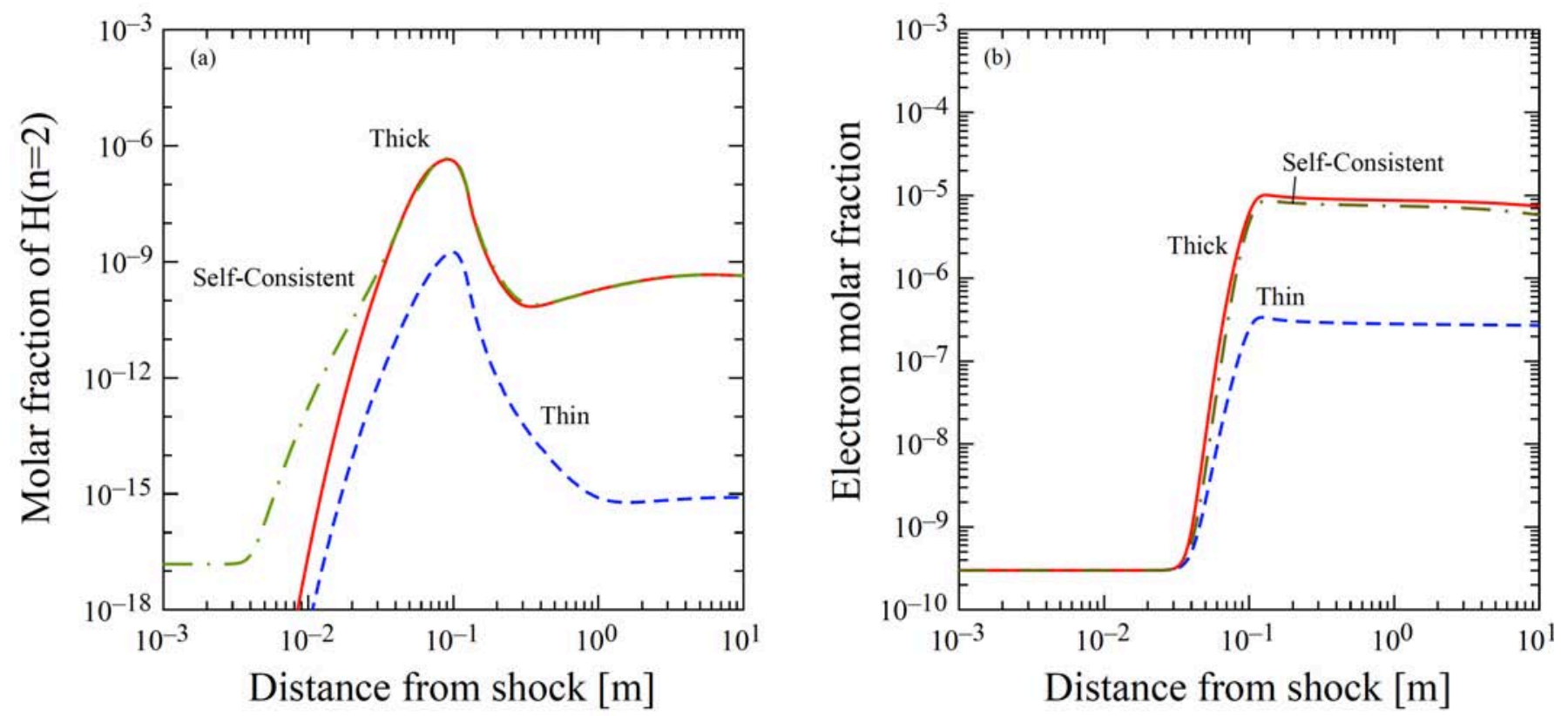

Fig. (8). (a) Molar fraction profiles of the first excited state of the $\mathrm{H}$ atom and (b) electron molar fraction profiles for the $M=20$ case.

resulting from the three models (SC, thick, thin). In the SC and thick case, the $\mathrm{H}(n=2)$ concentration tend to the same value for $x \geq 3 \mathrm{~cm}$, which is much higher compared to the optically thin case. On the other hand, at smaller distances the $\mathrm{H}(n=2)$ population predicted by the SC model is larger than the thick case due to the absorption of Lyman- $\alpha$ radiation generated in the emissive plasma region near the ionization maximum and moving towards the shock front. This effect, not present in both the plasma thick and thin cases, provides an additional excitation channel which can be important when the excitation by electron impact is slow due to very small electron concentrations. The molar fraction of electrons reported in Fig. (8b) shows a similar trend.
The internal distributions of $\mathrm{H}$ atoms resulting from the three optical models are reported in Fig. (9) as a function of the distance from shock, showing that already at $d=10 \mathrm{~cm}$ the thick and radiation coupled results coincide, being significantly higher than the corresponding thin distributions. The thermalization of the internal distribution happens at about $x=20.7 \mathrm{~cm}$ for thick and radiation coupled cases, when the electron number density is sufficiently high to promote electron impact processes. Moreover the higher concentration of excited states in the SC and thick case lead to stronger superelastic structures in the eedf compared to the optically thin case, as reported in Fig. (10). 

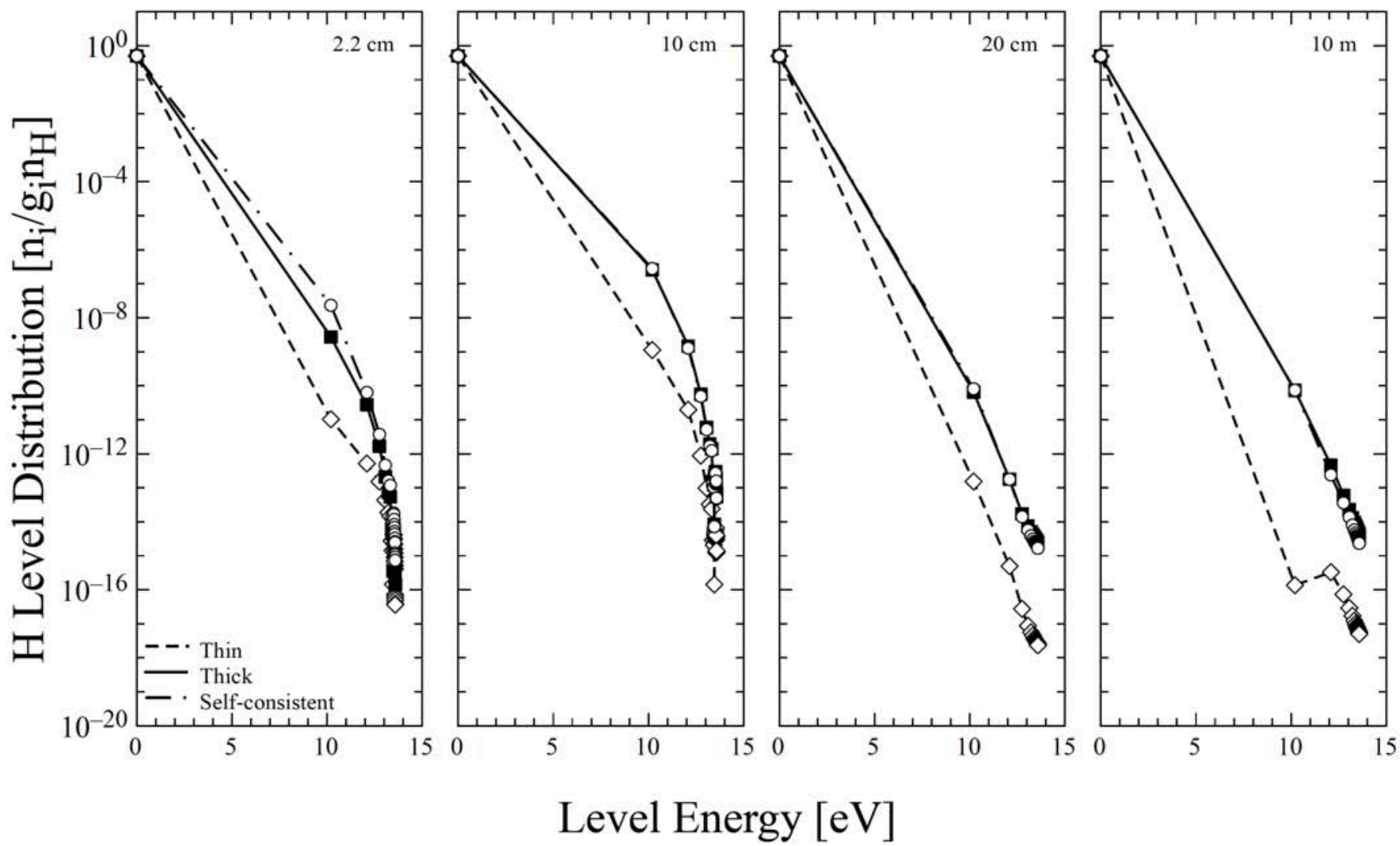

\section{Level Energy $[\mathrm{eV}]$}

Fig. (9). ISD of the $\mathrm{H}$ atom in the post-shock region for the $M=20$ case: comparison between optically thin, optically thick and selfconsistent calculations.

\section{CONCLUSION}

In this paper we have summarized the main features and possible applications of our collisional-radiative models for the study of shock waves and its radiation in hypersonic flows and atmospheric entry plasmas.

A description of an hybrid collisional-radiative model developed in the Laboratory of Radiative Gas Dynamics, Moscow has been presented. The model combines a state-tostate description of molecular electronic states with a multitemperature description of the remaining degrees of freedom, and solves the reactive Euler equations to determine flow parameters in the relaxation zone behind the shock front. The code can be applied to different gas mixtures, such as $\mathrm{N}_{2}-\mathrm{O}_{2}$, $\mathrm{CO}_{2}-\mathrm{N}_{2}$ and $\mathrm{CH}_{4}-\mathrm{N}_{2}$, using different kinetic models to study both non-equilibrium and equilibrium spectral intensities of radiation emitted by shock-heated gases. The code has already been used to analyze experimental shock tube results from different facilities around the world.

Next, an hybrid collisional-radiative model for ionized air, including a detailed scheme of electronically excited states of neutral $\mathrm{N}$ and $\mathrm{O}$ atoms has been described.

A one-dimensional Euler solver has been coupled with a collisional-radiative model and the radiation transport equation and internal distribution of atomic species derived in the state-to-state approach. The atomic state populations are found to depart from Boltzmann distributions because the high-lying bound electronic states are depleted. Moreover, the results emphasize the importance of fully (kinetic- radiation) coupled model, for a realistic description of the plasma, beyond thin and thick approximations.

Finally, a one-dimensional steady shock wave model coupling a comprehensive $\mathrm{CR}$ model of a $\mathrm{H}_{2} / \mathrm{He}$ plasma, a Boltzmann solver for the eedf and an RTE solver has been described. The model solves the steady state shock continuity equations, using the $\mathrm{CR}$ model to determine the non-equilibrium chemical composition and the distribution of atomic and molecular excited states. Electron-impact rate coefficients are directly calculated integrating the relevant cross-section over the actual eedf determined by the Boltzmann solver, while radiative rate coefficients are selfconsistently calculated using the local spectral intensity determined by the RTE solver. The model has been applied to study an high speed shock wave under conditions typical of a planetary entry in Jupiter's atmosphere. Dissipation of the shock energy happens mainly through dissociation of $\mathrm{H}_{2}$ molecules and ionization of the resulting $\mathrm{H}$ atoms, while $\mathrm{He}$ atoms are scarcely excited/ionized.

\section{CONFLICT OF INTEREST}

The authors confirm that this article content has no conflict of interest.

\section{ACKNOWLEDGEMENTS}

The research leading to these results has received funding from the European Community's Seventh Framework Programme [FP7/2007/2013] under grant agreement no. 242311. 



\section{Electron energy $[\mathrm{eV}]$}

Fig. (10). eedf in the post-shock region for the $M=20$ case: comparison between optically thin, optically thick and self-consistent calculations.

\section{REFERENCES}

[1] Surzhikov ST. Coupled radiation-gas dynamic model for sturdust aerothermodynamic data. AIAA 2010-4521

[2] Surzhikov ST. Radiative-gas dynamic model of martian descent Space vehicle. AIAA 2004-1355.

[3] Surzhikov ST. A study of the influence of kinetic models on calculations of the radiation-convective heating of a space vehicle in Fire - II flight experiment. Russian J Phys Chem B 2008; 2(5): 814-26.

[4] Grinstead JH, Wilder MC, Wright MJ, et al. Shock radiation measurements for mars aerocapture radiative heating analysis. AIAA 2008-1272.

[5] Cruden BA, Prabhu D, Martinez R, Le H, Bose D, Grinstead JH. Shock radiation measurements for Mars aerocapture radiative heating analysis. AIAA 2010-4508.

[6] Takayanagi H, Fujita K. Absolute radiation measurements behind strong shock wave in carbondioxide flow for mars aerocapture missions. AIAA 2012-2744.

[7] Brandis AM, Morgan RG, McIntyre TJ, Jacobs PA. Non-equilibrium radiation intensity measurements in simulated titan atmospheres. J Thermophys Heat Transf 2012; 24(2): 291-300.

[8] Kozlov PV, Romanenko YV, Shatalov OP. Radiation intensity measurement in simulated martian atmospheres on the double diaphragm shock tube. In: ESA, Eds. Proc. of $4^{\text {th }}$ International Workshop on Radiation of High Temperature Gases in Atmospheric Entry. ESA 2010; SP-689.

[9] Sharma SP, Ruffin SM, Gillespie WD, Meyer SA. Vibrational relaxation measurements in an expanding flow using spontaneous raman scattering. J Thermophys Heat Transf 1993; 7(4): 697-703.

[10] Siefert N, Hebner G. Shock velocity in a decaying nitrogen plasma. AIAA 2005-5381.

[11] Kudryavtsev NN, Kuznetsova LA, Surzhikov ST. Kinetics and nonequilibrium radiation of $\mathrm{CO}_{2}-\mathrm{N}_{2}$ shock waves. AIAA 2001-2728.

[12] Surzhikov ST, Sharikov I, Capitelli M, Colonna G. Kinetic models of non-equilibrium radiation for strong air shock waves. AIAA 2006-586.

[13] Dikalyuk AS, Surzhikov ST, Shatalov OP, Kozlov PV, Romanenko YV. Non-equilibrium radiation behind the strong shock waves in martian and titan atmospheres: numerical rebuilding of experimental data. AIAA 2012-0795.
[14] Huo MW, Panesi M, Magin ET. Ionization phenomena behind shock waves in High Temperature Phenomena in Shock Waves. Shock wave science and technology reference library - Springer 2011; 7: pp. 149-92.

[15] Panesi M, Magin T, Bourdon A, Bultel A, Chazot O. Analysis of the fire II flight experiment by means of a collisional radiative model. J Thermophys Heat Transf 2009; 23(2): 236-48.

[16] Panesi M, Magin T, Bourdon A, Bultel A, Chazot O. Electronic excitation of atoms and molecules for the fire II flight experiment J Thermophys Heat Transf 2011; 25(3): 361-74.

[17] Colonna G, Pietanza LD, D'Ammando G. Self-consistent collisionalradiative model for hydrogen atoms: Atom-atom interaction and radiation transport. Chem Phys 2012; 398: 37-45.

[18] Colonna G, D’Ammando G, Pietanza LD, Capitelli M. Self-consisten coupling of chemical, electron and radiation models for shock wave in Jupiter atmosphere. In: AIP Conf Proc 2012; 1501: pp. 1400-7.

[19] Capitelli M, Colonna G, D’Angola A. Fundamental aspects of plasma chemical physics: Thermodynamics. vol. 66, Atomic, Optical, and Plasma Physics. 1st ed. New York: Springer 2011.

[20] Colonna G, D’Angola A, Laricchiuta A, Bruno D, Capitelli M. Analytical expressions of thermodynamic and transport properties of the martian atmosphere in a wide temperature and pressure range. Plasma Chem Plasma Process 2013; 33(1): 401-31.

[21] D'Angola A, Colonna G, Gorse C, Capitelli M. Thermodynamic and transport properties in equilibrium air plasmas in a wide pressure and temperature range. Eur Phys J D 2008; 46(1): 129-50.

[22] Dikalyuk AS, Surzhikov ST. Numerical investigation of a model of non-equilibrium radiation behind a shock front in the martian atmosphere. Fluid Dyn 2013; 48(1): 123-40.

[23] Arsentiev IV, Loukhovitski BI, Starik AM. Application of state-to-state approach in estimation of thermally non-equilibrium reaction rate constants in mode approximation. Chem Phys 2012; 398: 73-80.

[24] Guy A, Bourdon A, Perrin MY. Derivation of a consistent multiinternal temperature model for vibrational energy excitation and dissociation of molecular nitrogen in hypersonic flows. AIAA 20130194.

[25] Kuznetsova LA, Surzhikov ST. Spectral radiation of shock waves and radiative models of diatomic molecules. AIAA 97-2564.

[26] Park C. Non-equilibrium Hypersonic Aerothermodynamics. Wiley 1990. 
[27] Park C. Review of chemical-kinetic problems of future NASA missions, I: Earth entries. J Thermophys Heat Transf 1993; 7(3): 38598.

[28] Park C, Howe JT, Jaffe RL, Candler GV. Review of chemical kinetic problems of future NASA mission. II Mars Entries. J Thermophys Heat Transf 1994; 8(1): 9-23.

[29] Losev SA, Makarov VN, Pogosbekyan MY. Model of the physicochemical kinetics behind the front of a very intense shock wave in air. Fluid Dyn 1995; 20(2): 299-309.

[30] Teulet P, Sarrette JP, Gomes AM. Calculation of electron impact inelastic cross sections and rate coefficients for diatomic molecules. Application to air molecules. J Quant Spectrosc Ra 1999; 62: 549-69.

[31] Park C. Rate parameters for electronic excitation of diatomic molecules I. Electron-Impact Processes. AIAA 2008-1206.

[32] Starik AM, Titova NS, Arsentiev IV. Comprehensive analysis of the effect of atomic and molecular metastable state excitation on air plasma composition behind strong shock waves. Plasma Sources Sci Technol 2010; 19: 015007.

[33] Sharipov AS, Starik AM. Kinetic mechanism of $\mathrm{CO}-\mathrm{H}_{2}$ system oxidation promoted by excited singlet oxygen molecules. Combust Flame 2012; 159(1): 16-29.

[34] Cartwright DC. Rate coefficients and inelastic momentum transfer cross sections for electronic excitation of $\mathrm{N}_{2}$ by electrons. J Appl Phys 1978; 49(7): 3855-62.

[35] Park C. Rate parameters for electronic excitation of diatomic molecules II. Heavy particle impact processes. AIAA 2008-1446.

[36] Harrison S, Tennyson J. Electron collisions with $\mathrm{CN}$ radical: bound states and resonances. J Phys B 2012; 45: 035204.

[37] Cherniy GG, Losev SA. Physical-chemical processes in gas dynamics: reference book. Moscow: MSU publishing 1995.

[38] Zeidler KT, Koester D. On the "just overlapping line approximation" for molecular absorption. Astron Astrophys 1982; 113: 173-5.

[39] Zalogin GN, Kozlov PV, Kuznetsova LA, et al. Radiation excited by shock waves in a $\mathrm{CO}_{2}-\mathrm{N}_{2}-\mathrm{Ar}$ mixture: experiment and theory. Tech Phys 2001; 46(6): 654-61.

[40] Johnston CO. Non-equilibrium Shock-layer radiative heating for earth and titan entry. Virginia; Virginia Polytechnic Institute and State University 2006.

[41] Zeldovich Y, Raizer Y. Physics of shock waves and high temperature hydrodynamic phenomena. Berkley Square House, London: Academic Press Inc 1966.

[42] Park C. Non-equilibrium hypersonic aerothermodynamics. New York: John Wiley and Sons 1990.

[43] Panesi M, Jaffe RL, Schwenke D, Magin ET. Rovibrational internal energy transfer and dissociation of $\mathrm{N}_{2}-\mathrm{N}$ system in hypersonic flows. J Chem Phys 2013; 138: 44312.

[44] Magin TE, Panesi M, Bourdon A, Jaffe R, Schwenke D. Uniform rovibrational collisional coarse-grain model for internal energy excitation and dissociation of molecular nitrogen. J Chem Phys 2011; 398: 90-5.

[45] Schwenke D. Dissociation cross-sections and rates for nitrogen. VKI LS 2008, Non-equilibrium gas dynamics, from Physical models to hypersonic flights - Rhode-Saint-Genése, Belgium 2008.

[46] Martin WC, Fuhr JR, Kelleher DR, Masgrove A, Sugar J, Wiese WL. NIST atomic spectra database (version 2.0). Avaliable from: http://physicsnistgov/asd2 Gaihersburg 1999.

[47] Gurvich LV, Veyts IV, Alcock CB. Thermodynamic properties of individual substances. Hemisphere Publishing Corporation 1989; 1.

[48] Bultel A, Chéron BG, Bourdon A, Motapon O, Schneider IF. Collisional-radiative model in air for earth re-entry problems. Phys Plasmas 2006; 13(4): 11.
[49] Teulet P, Sarrette JP, Gomes AM. Collision-radiative modeling of oneand two temperature air and air-sodium plasmas at atmospheric pressure with temperatures of 2000 - 12000 K. J Quant Spectrosc Ra 2001; 1: 70-159.

[50] Sarrette JP, Gomes AM, Bacri J, Laux CO, Kruger CH. Collisionalradiative modelling of quasi-thermal air plasmas with electronic temperatures between 2000 and 13,000 K-I. $\Theta_{e}>4000 \mathrm{~K}$. J Quant Spectrosc Ra 1995; 53(2): 125-41.

[51] Colonna G, Capitelli M. The influence of atomic and molecular metastable states in high-enthalpy nozzle expansion nitrogen flows. J Phys D 2001; 34: 1812.

[52] Colonna G, Tuttafesta M, Capitelli M, Giordano D. Non-arrhenius NO formation rate in one-dimensional nozzle airflow. J Thermophys Heat Transf 1999; 13(3): 372-5

[53] Colonna G, Capitelli M. Self-consistent model of chemical, vibrational, electron kinetics in nozzle expansion. J Thermophys Heat Transf 2001; 15(3): 308-16.

[54] Borghi CA, Carraro MR, Cristofolini A, et al. Magneto-hydrodynamic interaction in the shock layer of a wedge in a hypersonic flow. IEEE Trans Plasma Sci 2006; 34: 2450-63.

[55] Colonna G, Capitelli M. Boltzmann and master equations for magnetohydrodynamics in weakly ionized gases. J Thermophys Heat Transf 2008; 22(3): 414-23.

[56] Armenise I, Capitelli M, Colonna G, Koudriavtsev N, Smetanin V. Non-equilibrium vibrational kinetics during hypersonic flow of a solid body in nitrogen and its influence on the surface heat-flux. Plasma Chem Plasma Process 1995; 15(3): 501-28.

[57] Colonna G, Capitelli M. Electron and vibrational kinetics in the boundary layer of hypersonic flow. J Thermophys Heat Transf 1996; 10(3): 406-12.

[58] Giordano D, Bellucci V, Colonna G, Capitelli M, Armenise I, Bruno C. Vibrationally relaxing flow of $\mathrm{N}_{2}$ past an infinite cylinder. J Thermophys Heat Transf 1997; 11(1): 27-35

[59] Capitelli M, Armenise I, Bruno D, et al. Non-equilibrium plasma kinetics: a state-to-state approach. Plasma Sources Sci Technol 2007; 16: S30-44.

[60] Colonna G, Pietanza LD, Capitelli M. Coupled solution of a timedependent collisional-radiative model and Boltzmann equation for atomic hydrogen plasmas: possible implications with LIBS plasmas. Spectrochim Acta B 2001; 56: 587-98.

[61] Fujimoto T. A collisional-radiative model for helium and its application to a discharge plasma. J Quant Spectrosc Ra 1979; 21: 439-55.

[62] Goto M. Collisional-radiative model for neutral helium in plasma revisited. J Quant Spectrosc Ra 2003; 76(3-4): 331-44.

[63] Ralchenko Y, Janev RK, Kato T, Fursa DV, Bray I, de Heer FJ. Electron-impact excitation and ionization cross sections for ground state and excited helium atoms. Atom Data Nucl Data 2008; 94: 603-22.

[64] Kramida A, Ralchenko Y, Reader J, NIST ASD Team. NIST atomic spectra database (version 5.0). Gaithersburg, MD 2012

[65] Team O. The Iron Project - The Opacity Project 2012. Available from: http: //cdsweb.u-strasbg.fr/topbase/topbase.html

[66] Mihalas D, Mihalas BW. Foundations of radiation hydrodynamics. Dover Publications 1999.

[67] Drawin HW. Influence of atom-atom collisions on the collis-ionalradiative ionization and recombination coefficients of hydrogen plasmas. Zeitschrift fur Physik 1969; 225(5): 483-93.

[68] Drawin HW, Emard F. Atom-atom excitation and ionization in shock waves of the noble gases. Phys Lett A 1973; 43(4): 333-5.

[69] Capitelli M, Colonna G, Pietanza LD, D'Ammando G. Coupling of radiation, excited states and electron energy distribution function in non-equilibrium hydrogen plasmas. Spectrochim Acta Part B 2013; 8384: 1-13. 